\title{
High Energy Physics Astro Particle Experiments to Improve the Radiation Health Risk Assessment for Humans in Space Missions
}

\author{
A. Bartoloni, ${ }^{a}$, , G. Della Gala ${ }^{a, b}$, A.N. Guracho ${ }^{a}$, G. Paolani $a, b$, M. Santoro ${ }^{a, b}$ L. \\ Strigari $a, b$, S. Strolin $a, b$ \\ a INFN Sezione di Roma, \\ Ple Aldo Moro n.2, Rome, Italy \\ b IRCCS University Hospital of Bologna, \\ Via Massarenti 9, Bologna, Italy \\ E-mail: alessandro.bartoloni@romal.infn.it, \\ giuseppe.dellagala@aosp.bo.it, aboma.guracho@romal.infn.it, \\ lidia.strigarieaosp.bo.it, miriam.santoro@aosp.bo.it, \\ lidia.strigarieaosp.bo.it, silvia.strolineaosp.bo.it
}

In the near future, all the space agencies are working to restart the human exploration of space outside the Low Earth Orbit (LEO). Crewed space missions in this and the next decade will see the presence of humans on the Moon and Mars surface. One of the main showstoppers to be investigated for safe exploration and colonisation is the biological effects of ionising radiation that can compromise the health of astronauts/space workers. In this vital task, a principal role could be done by the astroparticle experiments presently operating in space. Such experiments are a source of information crucial to improving the knowledge of radiobiology effects in space. In this talk, a review of the past and present astroparticle experiments will be presented and will highlight some of the possible contributions and improvements in the space radiobiology research field.

The European Physical Society Conference on High Energy Physics (EPS_HEP2021)

26-30 July 2021

Online conference, jointly organized by Universität Hamburg and the research center DESY

*Speaker

(C) Copyright owned by the author(s) under the terms of the Creative Commons

Attribution-NonCommercial-NoDerivatives 4.0 International License (CC BY-NC-ND 4.0). 


\section{Introduction}

Cosmic rays (CR) approaching our planet interact with the Earth's magnetic field, and atmosphere and such interaction protect humans living on the Earth's surface. The magnetosphere rejects most of the particles (99\%) while the rest lose most of their energy going through the atmosphere before reaching the Earth's surface [1]. In particular, the geo magnetosphere stops/deflects $99.9 \%$ of charged particles while the Earth's atmosphere is equivalent to a metal shielding 1 meter thick. Completely different is the situation in space where the CR interacting with the human body release some energy and can be dangerous for human health. In this regard, this is one of the main concerns for safe space exploration as planned for the coming years by all the national space agencies $[2,3]$. In this context, all the different components of space radiation have been extensively studied and measured during the last decades by several astroparticle experiments operating in space, and the information contained in the data taken by such experiments can be used to improve the radiation health risk assessment for humans in space missions.

\section{Astro Particle experiment in space}

In the last two decades, many astroparticle experiments have been built and deployed in space to investigate many open questions in fundamental physics and cosmology, for example, the dark matter and dark energy existence and composition or the existence of primordial antimatter. A particular class of experiments, the Cosmic Ray Detectors (CRD), is designed to produce a complete inventory of charged particles and nuclei in CR since the knowledge of this information is crucial to solving the above physics open problems.

\subsection{Principal Operating Cosmic Ray Space Detectors}

By now, several CRDs are doing precise measurements of CR components in the energy region from few $\mathrm{KeV}$ to hundreds of $\mathrm{TeV}$, and in particular, we discuss in this paper the following five:

- The Alpha Magnetic Spectrometer (AMS-02) is a high-energy particle physics experiment in space designed to measure cosmic rays (CRs). The primary purposes of the experiment are the indirect search of dark matter from its annihilation products, the search of relic anti-matter and the precise measurement of all CRs species spectra, and their variation in time for the precise estimation of radiation doses for space exploration. The AMS-02 spectrometer consists of a permanent magnet and several instruments (subdetectors) and in particular, a Silicon Tracker, a Time of Flight (TOF), a Ring Image Cherenkov Counter (RICH), and Electromagnetic Calorimeter (ECAL), an Anticoincidence Counter (ACC) and a Transition Radiation Detector (TRD). AMS-02 provides excellent particle identification capabilities. It measures the charge of the traversing particle independently in Tracker, RICH, and TOF subdetectors. The TOF, TRD, and RICH sub-detector measures the particle velocity. AMS02 was launched and installed on the International Space Station (ISS) in May 2011 and has been continuously operating since then [4-7].

- the Dark Matter Particle Explorer (DAMPE): it is a satellite-based space mission whose main purpose is the detection of cosmic electrons and photons up to energies of $10 \mathrm{TeV}$. The DAMPE instruments have also the capability to measure the fluxes and the elemental 
composition of the galactic cosmic rays' nuclei up to $100 \mathrm{TeV}$. It is in data taking since 2015 and consists of: a double layer of Plastic Scintillator Detector; a Silicon-tungsten Trackerconverter; an electromagnetic calorimeter and a Neutron Detector. In the first's years of operations DAMPE has collected and send to the earth more than 6 billion of cosmic ray events [8].

- the CALorimetric Electron Telescope (CALET): it is in operation since 2015 on the external platform of the Japanese experimental module (KIBO/JEM) by the ISS. The instrument is optimized to precisely study the properties of extreme energy cosmic electrons up to dozens of $\mathrm{TeV}$. It is also capable of measuring the relative composition and abundance of nuclei coming from space, from protons to the heaviest elements up to $Z=40$. In the first three years of operation CALET has collected and send to the earth more than 1.8 billion of cosmic rays' events [9].

- The Cosmic Ray Energetic and Mass for the International Space Station (ISS-CREAM): it was successfully installed and activated on the Japanese Experiment Module Exposed Facility as an attached payload in 2017. The instrument is configured with complementary particle detectors capable of measuring elemental spectra for $Z=1$ up to $Z=26$ nuclei in the energy range from 1 up to $1000 \mathrm{TeV}$; as well as electrons at multi-TeV energies add the CREAM instrument [10].

- the Advanced Composition Explorer (ACE) is a satellite-based space mission that started its operation on 1998 with the aim to observe particles of solar, interplanetary, interstellar, and galactic origins, spanning the energy range from solar wind ions to galactic cosmic ray nuclei. It is located at the L1 Lagrange point, about 1.4 million kilometres from the Earth. Thanks to the multiple instruments on board ACE can measure particle and nuclei elements up to $Z=30$ in an energy range up to hundreds of $\mathrm{MeV}$. The end of its operation is forecast not before the 2024 [11].

Another important CRD, no more in operation, was the Payload for Antimatter Matter Exploration and Light-nuclei Astrophysics (PAMELA) that starting its data taking in 2006 and ends its operations in 2016, producing in this period accuracy measurements of the cosmic ray components (particle and light nuclei up to $Z=6$ ) [12].

\subsection{Cosmic ray detectors operations and measurements}

The characteristics of the operations of the CRD space missions that lasts for several years are optimal to use the huge amounts of data (on average a CRD registers more than a billion of CR events per year, i.e. AMS02 have registered since the start of data taking in 2011 more than 190 billion of events), taken to improve our knowledge of the IR health effects on humans in space and can be summarized in the following aspects[13]:

- Complete CR components identification: They can measure the light components like electrons and protons and the nuclei from the light one like the Helium up to heavier like the Iron $(Z=25)$.

- High Energy Range Spectra: As shown in figure 1, the data taken from the operating CRDs ranges from a few $\mathrm{MeV}$ up to hundreds of $\mathrm{TeV}$. 
- CR Solar Modulation: one of the most important aspects to be evaluated is the differences in IR exposition due to the interference of the solar activities and cycles with the GCR part of the space radiation. In this regard, the CRDs took data during cycles 23 and 24 and some will continue for the 25 th.

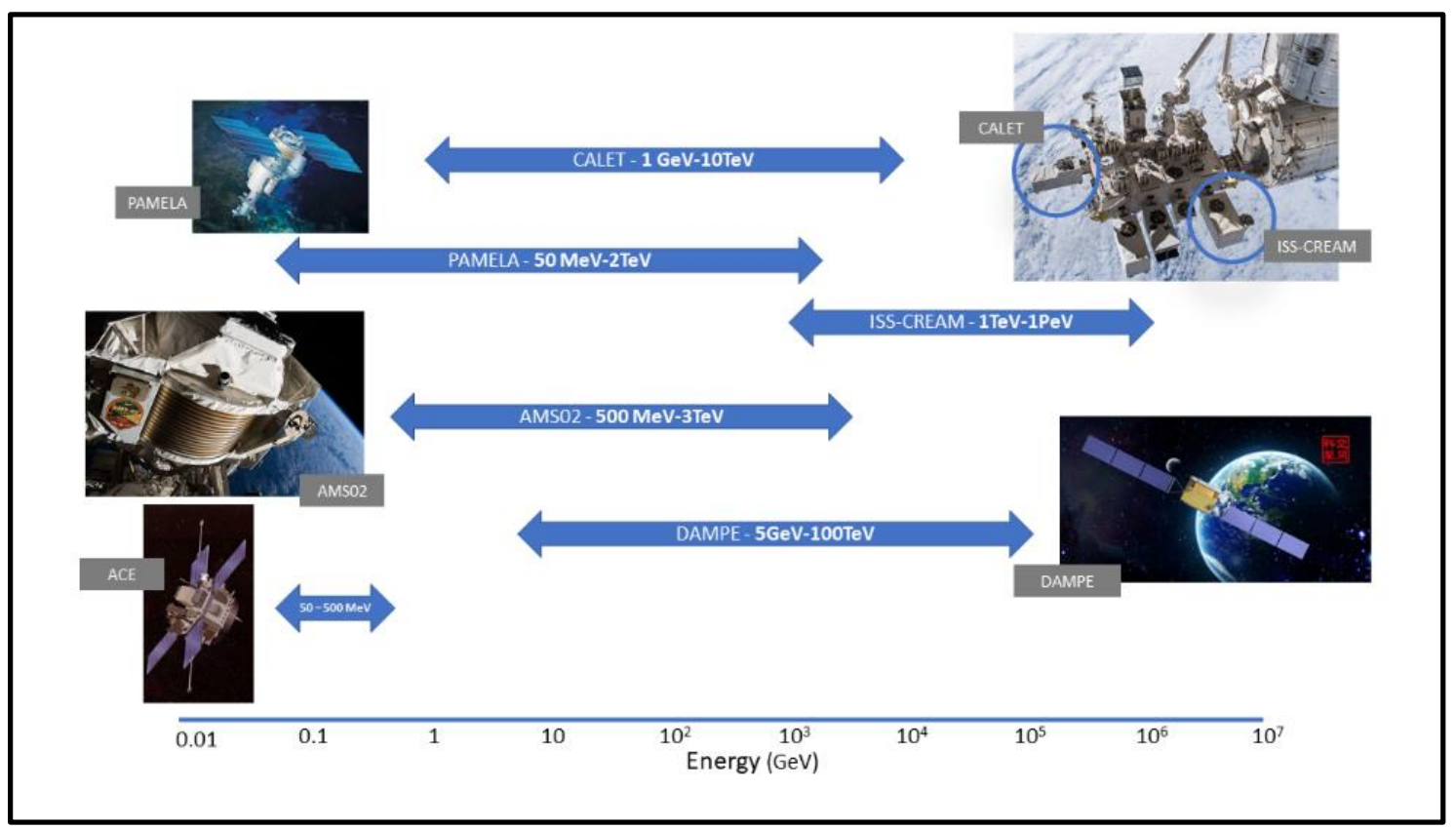

Figure 1: Measurement's energy range of the Cosmic Ray Detectors

\subsection{Galactic Cosmic Ray (GCR) sensitivity analysis: A case study on AMS02 capability}

In 2019 we identified which components of the CR are of interest for the computation of possible risks associated with the crewed exploratory space missions in LEO and BLEO scenarios. In this regard, using as reference some existing space radiation sensitivity studies [14], we also recognized that they correspond with the data taken by the CRDs operating in space, and in particular, the energy range crucial for the risk assessment mostly correspond to the capability of measurements of the AMS02 detector [15]. This study was the first step of an analysis focused on identifying possible improvements from the CDR data to the radiation health risk assessment for human space missions [16].

\section{Improve the Radiation Health Risk Assessment for Humans in Space Missions}

In the second step, we perform a literature search of published dose-effect relationships identifying the reported endpoints from space missions, including acute and late effects, published in a separated manuscript [17].

In this study, we highlight the significant improvements in the risk assessment capability thanks to the possibility to have information in energy ranges never explored before and in the complete species of CR - from elementary particles (electrons, protons) to light and heavy nuclei (Helium to Iron and beyond) - that could be of concerns in space missions. Figure 2. shows the identified possible improvements. 


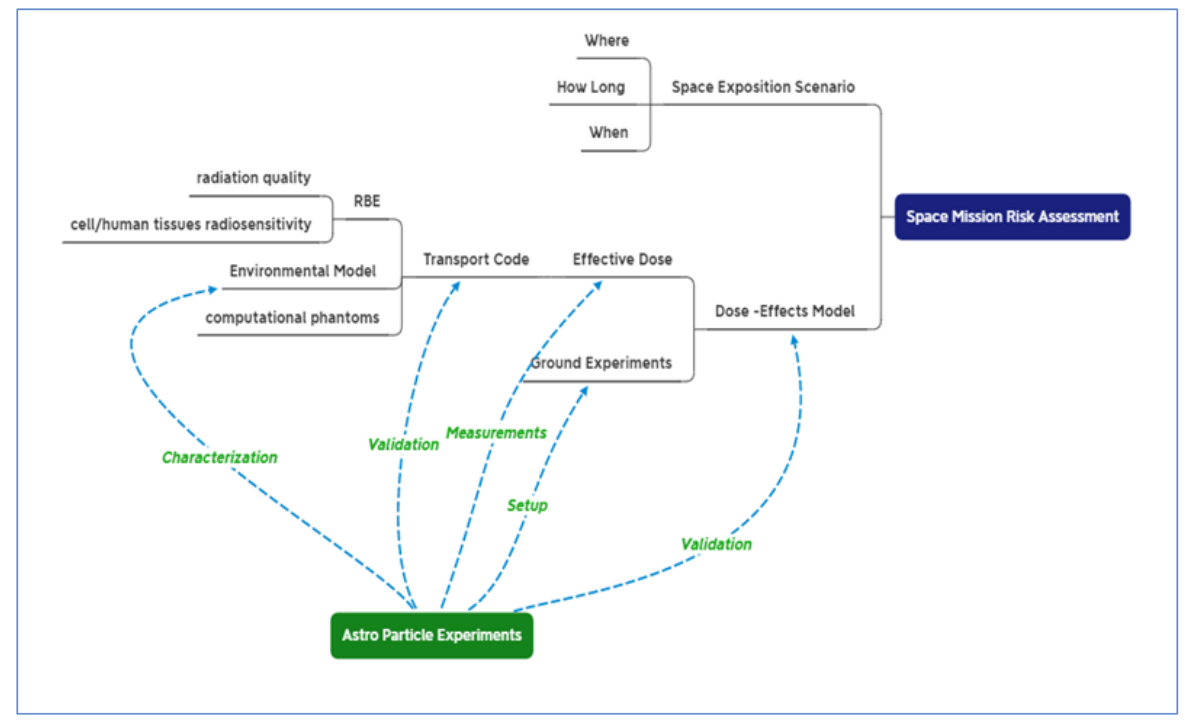

Figure 2: Human Health Risk Assessment in exploratory space missions: possible improvement using Astro Particle experiments operating in space.

\subsection{Other possible improvements}

There are many other aspects of IR health effects on humans that could be investigated prior to long-term space missions outside the protection of the Earth's geomagnetic sphere will begin and that can benefit of the information contained in the CRD data. Among the others:

- The so called "non target effects" to be intended as the damage generated in tissue not directly irradiated. This topic is most unknow due to the scarcity of data with animal models for tissues that dominate human radiation cancer risk

- The individual radiosensitivity, that it is very important when the number of people travelling/working in space will be orders of magnitude higher than the actual one

\section{Conclusions and acknowledgement}

In the coming years there will be a great interest for space human mission non only to explore but also for a permanent presence of humans outside the geo-magnetosphere. Possible exposition to space radiation is a main concern and the first one showstopper in many human exploration scenarios. In this context a great benefit could derive from the huge amount of data acquired since more than a decade by the Astro particle experiments operating in space. Such data contrains crucial information on the composition and the radiation quality of the CR in LEO and BLEO environments and could be used to improve important topics related to the human health effects due to ionizing radiation expected in space during the planned space exploration missions. In particular, improvements of the efficacy of space radiobiology dose-effects models is possible while many other topics till not Completely investigated (non-targeted effects, individual radio sensitivity, ...) could be further investigated.

We gratefully acknowledge the strong support from the AMS collaboration, from the INFN Scientific Committee 2 (CSN2) and from the Italian Space Agency (ASI) within the agreement ASI-INFN n. 2019-19-HH.0. 


\section{References}

[1] C. Leroy, P. Rancoita, Principles of Radiation Interaction in Matter and Detection. World Scientific, 2016.

[2] L. Walsh et al, Research plans in Europe for radiation health hazard assessments in exploratory space missions, Life Sciences in Space Research 21 (2019) 73-82. DOI: 10.1016/j.lssr.2019.04.002

[3] International Space Exploration Coordination Group (ISCECG), Global Exploration RoadmapLunar Surface Exploration Scenario, August 2020 update

[4] M. Aguilar et al., The Alpha Magnetic Spectrometer (AMS) on the international space station: Part II - Results from the first seven years, Phys.Rept. 894 (2021), 1-116, DOI: 10.1016/j.physrep.2020.09.003

[5] AMS Collaboration, M. Aguilar (Madrid CIEMAT) et al., Properties of Iron Primary Cosmic Rays: Results from Alpha Magnetic Spectrometer, Jan 29, 2021, Published in: Phys.Rev.Lett. 126 (2021) 8, 081102, DOI:10.1103/PhysRevLett.126.041104

[6] AMS Collaboration, M. Aguilar (Madrid CIEMAT) et al., Properties of heavy Secondary Fluorine Cosmic Rays: Results from Alpha Magnetic Spectrometer, Jan 29, 2021, Published in: Phys.Rev.Lett. 126 (2021 4, 041104, DOI:10.1103/PhysRevLett.126.081102

[7] AMS Collaboration, M. Aguilar (Madrid CIEMAT) et al., Properties of a New Group of Cosmic Nuclei: Results from the Alpha Magnetic Spectrometer on Sodium, Aluminum, and Nitrogen, Jul 7 , 2021, Phys.Rev.Lett. 127 (2021) 15, 159901 (erratum), DOI:10.1103/PhysRevLett.127.159901

[8] A. De Benedittis for the DAMPE Collaboration, The DAMPE experiment: performance and results, in proceedings of EPS-HEP 2019, DOI: 10.22323/1.364.0030

[9] CALET Collaboration Y. Asaoka et al, The CALorimetric Electron Telescope (CALET) on the International Space Station: Results from the First Two Years on Orbit, J.Phys.Conf.Ser. 1181 (2019), DOI: 10.1088/1742-6596/1181/1/012003

[10] E.S. Seo et al (ISS-CREAM collaboration), Cosmic Ray Energetics and Mass for the International Space Station (ISS-CREAM), proceedings of the ICRC2019, DOI:10.22323/1.358.0137

[11] E. Stone, A. Frandsen, R. Mewaldt,. et al., The Advanced Composition Explorer, Space Science Reviews 86 (1998) 1-22, DOI: 10.1023/A:1005082526237

[12] O. Adriani, et al, Ten years of PAMELA in space, Rivista del Nuovo Cimento, 10 (2017) 473-522. DOI: $10.1393 / \mathrm{ncr} / \mathrm{i} 2017-10140-\mathrm{x}$

[13] A. Bartoloni, S. Strolin, L. Strigari, 2020 Virtual IEEE Nuclear Science Symposium and Medical Imaging Conference Presentazione standard di PowerPoint (infn.it) (accessed 10.10.21)

[14] T. Slaba, S. Blatting, GRC environmental model I: Sensitivity analysis for GCR environments, Space Weather 12:4 (2014) 217-224, DOI: 10.1002/2013SW001025

[15] A. Bartoloni, L. Strigari, AMS02 e radiobiologia nello spazio, in proceedings of the Annual Conference of the Italian Physical Society (SIF) L'Aquila 27/09/2019.

[16] A. Bartoloni, L. Strigari, Can high energy particle detectors be used for improving risk models in space radiobiology?, in proceedings of the Global Space Exploration Conference 2021 (GLEX2021) Jun 2021.

[17] L.Strigari, S. Strolin, A. G. Morganti and A. Bartoloni, Dose-Effects Models for space radiobiology: an overview on dose effect relationships, to be published on Frontiers in Public Health- Radiation and Health, DOI: 10.3389/fpubh.2021.733337 